\title{
Problem-solving in open environments
}

\author{
Santiago Macho-Gonzalez and Boi Faltings \\ Artificial Intelligence Laboratory (LIA), \\ Swiss Federal Institute of Technology (EPFL), \\ IN-Ecublens, CH-1015 Ecublens, Switzerland \\ http://liawww.epfl.ch/ \\ E-mail: boi.faltings|santi.machodepfl.ch
}

\begin{abstract}
With the increasing use of the internet, many problemsolving tasks such as resource allocation, scheduling, planning, and configuration pose themselves in an open setting involving multiple participants. Existing search-based problem-solving techniques are based on the closed-world assumption and require that all options be collected before problem-solving can start. This approach of turning the web into a virtual database often leads to gathering much more information than necessary to solve the problem.

We consider constraint satisfaction as the most successful practical problem solving technique, and define open constraint satisfaction where values are incrementally gathered during problem solving. We present several algorithms for open constraint satisfaction and show that on random problems, they require gathering significantly less values. We then extend the technique to constraint optimization using the fuzzy CSP model, and show that here too great effciency gains can be achieved by coupling problem solving and information gathering.
\end{abstract}

\section{Problem-solving in open systems}

Constraint satisfaction has found wide applicability for problem-solving, in particular for resource allocation, scheduling, planning and configuration. Efficient techniques have been developed for solving such problems in closed environments where all options and constraints are known and fixed from the beginning.

With the arrival of the internet, there is an increasing desire to automate problem-solving for scenarios that are distributed over a network of agents. This can be addressed with existing tools by adding an information gathering stage where options and constraints are collected from the partic- ipants. The problem can then be solved as before using a centralized constraint solver. Work in the database community has addressed the issue of how to make a distributed network of information sources appear as a single database, see for example Infomaster ([1]) or Infosleuth ([2]).

However, this approach has the drawback that it usually collects more information than necessary: it is usually not necessary to know all options in order to find a solution to the problem. Even for optimization, under some assumptions it is possible to compute the optimal solution without knowing the complete variable domains. The gains that can be achieved depend on how difficult the problem is: if there are many solutions, it is usually possible to find one with only few values, but if there is no solution, at least for some variables all values must be examined before this can be concluded.

The following example illustrates the point: consider planning a trip involving air travel and a hotel in city X. If we find out that all hotels in $\mathrm{X}$ are solidly booked, the problem solver has to find another city $\mathrm{Y}$ that both has available hotel space and suitable transportation schedules. If information gathering is separate from problem-solving, such contingencies would have to be allowed for in any query thus collecting a lot more information than is normally necessary. Driving information gathering by the problem solver makes it possible to collect the right information only when it is required.

In this paper, we present techniques for coupling problem-solving with information gathering to optimize the number of values that must be queried. The techniques will help to minimize the amount of information that must be gathered to solve problems. The gains can be particularly interesting in situations such as supply chains where problem-solvers are chained so that the results of some become the values used in others. In this case, the gains multiply and can produce truly dramatic improvements in the 
message traffic that is required to solve the problem.

We then extend the technique to constraint optimization using the fuzzy CSP model. This extension is based on the assumption that information sources always return the most preferred values first. We show how this makes it possible to gurantee optimality without obtaining all values, and show two algorithms that provide significant efficiency gains over the database approach.

\section{Related work}

Information integration has been studied by database researchers for a long time ([3]). With the increased use of networking, and particularly the internet, projects such as TSIMMIS ([4]), Infomaster ([1]), and others have addressed dynamic integration of information in order to make the distributed network of informationsources appear as a single database. InfoSleuth ([2]) has built a more elaborate infrastructure using information agents that not only integrate information, but can also provide proactive services such as subscriptions and change notifications. Our work assumes the availability of such techniques in order to transform the results of information sources into the format required for problem solving.

Another important topic is how to locate information sources that are capable of answering queries. In this paper, we assume an ontology-based classification similar to that of [5]. However, the matchmaking process can get significantly more complex. The Information Manifold ([6]) has shown that query planning techniques can be used to combine information from different information sources to obtain arbitrary relations, and this would be a useful extension to our work.

Decker and Sycara ([7]) investigate the efficiency of middle-agent systems for matchmaking, Techniques such as LARKS ([8]) show that much more complex matchmaking than ontology-based classification is possible, and it would be possible to derive such criteria from the problem-solver as well. While we have not seen other work that integrates information gathering and problem-solving in a similar way as we do, the KRAFT project ([9]) has used constraints as a formalism for representing and integrating data, but does not address integration of information gathering with problem-solving.

Recently, researchers in information retrieval have paid more attention to driving information retrieval from the task that users are trying to solve. Systems such as Watson and I2I ([10]) and just-in-time information retrieval ([11]) automatically retrieve information from databases, mail archives and other information sources by matching it with keywords that occur in the current activity of the user - for example, a document being prepared.

In the constraint satisfaction (CSP) community, there are

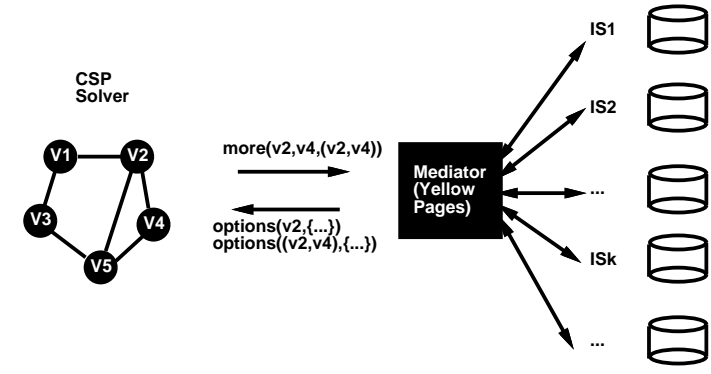

Figure 1. Elements of an open constraint satisfaction problem

algorithms for dynamic CSP [12] that address dynamic additional and removal of constraints, but assume that the space of value is completely known and fixed. Algorithms for distributed CSP [13] focus on asynchronous and distributed solving, but assume that both constraints and values are fixed during execution.

\section{Open Constraint Satisfaction Problems (OCSP)}

We consider the setting shown in Figure 1 which reflects the important elements that occur in an open setting. The problem-solving process is modelled abstractly as the solution of a constraint satisfaction problem. The choices that make up variables domains and relations of the CSP are distributed throughout an unbounded network of information servers $I S_{1}, I S_{2}, \ldots$, and accessed through a mediator ([14]).

More precisely, the CSP is an open constraint satisfaction problem, defined formally as follows:

Definition $1 A n$ open constraint satisfaction problem (OCSP) is a possibly unbounded, partially ordered set $(\operatorname{CSP}(0), \operatorname{CSP}(1), \ldots)$ of constraint satisfaction problems, where $\operatorname{CSP}(i)$ is defined by a tuple $<X, D(i), C>$ where

- $X=\left\{x_{1}, x_{2}, \ldots, x_{n}\right\}$ is a set of $n$ variables,

- $D(i)=\left\{d_{1}(i), d_{2}(i), \ldots, d_{n}(i)\right\}$ is the set of domains for $\operatorname{CSP}(i)$, with the condition $(\forall k) d_{k}(i) \subseteq d_{k}(i+1)$, and $d_{k}(0)=\{\}$ for all $k$.

- $C=\left\{\left(v_{i}, v_{j}\right),\left(v_{k}, v_{l}\right), \ldots\right\}$ is a set of $m$ constraints, given by the ordered sets of variables they involve and the value combinations they allow.

A solution of the OCSP is a combination of value assignments to all variables such that for some CSP $(i)$, each value belongs to the corresponding domain and all constraints are satisfied. 
Note that we assume that all constraints are binary, and that only variable domains can change over time. If the original problem does not fit this restriction, constraints that are nonbinary or that are incrementally discovered can be turned into a variable using hidden variable encoding ([15, 16]) which has as values the tuples allowed by the constraint and is linked to variables involved in the original constraint by new binary constraints that enforce equality between the variable values and the corresponding elements of the tuple.

Solving an OCSP requires an integration of search and information gathering. It typically starts from a state where all domains are empty, and the first action is to find values that fill the domains and allow the search to start. As long as the available information does not define enough values to make the CSP solvable, the problem solver initiates further information gathering requests to obtain additional values. The process stops as soon as a solution is found.

The problem solver accesses information on the network through a mediator ([14]). The mediator is a broker (as defined in [7]) that has to fulfill two important functions:

- a directory (yellow pages) for locating information servers that can supply values that can be used in the CSP that is being solved.

- an integrator that reformulates information from the servers to fit a format required by the CSP.

In order to be generally useable, the mediator must be compatible with any CSP that a user might want to solve. This is achieved through ontologies that define the meaning of CSP variables and values:

Definition 2 An ontological grounding of an open $C S P<$ $X, D(i), C>$ is a tuple $<O, M_{X}, M_{D}>$ where:

- $O=\left\{o_{1}, \ldots, o_{m}\right\}$ is a set of ontologies,

- $M_{X}$ is a mapping $X \rightarrow O \times C(O) \times P(O)$ that maps each variable $x_{j}, j=1 . . n$ into a tuple $<o_{j}, c_{k}, p_{l}>$ which means that $x_{j}$ models the property $p_{l}$ (sometimes called relation in the literature) of concept $c_{k}$ in ontology $o_{j}$. Note that the mapping need not be bijective, i.e. there can be several variables that map to the same concept and property.

- $M_{D}$ is a mapping $X \rightarrow O \times 2^{C(O)}$ that maps each variable $x_{j}, j=1 . . n$ into a tuple $<o_{j},\left\{c_{1}, . ., c_{k}\right\}>$ that defines the possible values that could be part of the variable's domain.

The ontological grounding allows the problem solver to define what information is required to fill the different aspects of the problem. It is fixed throughout the problem-solving, and communicated to the mediator either once at the beginning or with each request.

\section{Matching CSP variables to information sources}

The connection between CSP and information sources is based on its ontological grounding. We make the following assumptions:

- every query for more values refers to one ontology only.

- all information sources relevant to an ontology are classified according to this ontology, i.e. an information source that returns values for properties or relations of a concept is indexed under that concept.

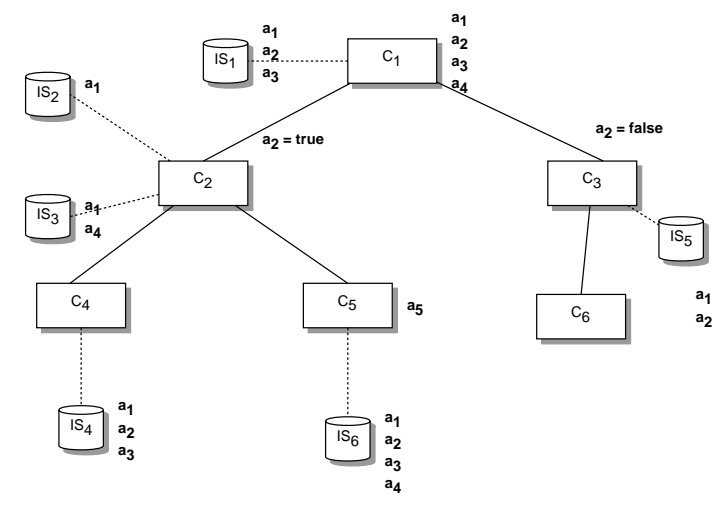

Figure 2. Indexing information sources (IS) with an ontology.

We use the technique of [5] to index information sources according to an ontology. Figure 2 shows an example. Several of the concepts have associated information sources (IS) that hold values and value combinations of their properties.

In the simplest algorithm, we only consider information sources that are directly indexed by the property $\mathbf{p}$ and concept $\mathbf{c}$ given in the query to the mediator. The mediator then accesses the information sources in a random order, each time returning all values obtained from the next source.

An improvement can be obtained by also noting how many values of each property each information source contains, and accessing those that provide many values first.

\section{Algorithms for solving OCSP}

We now consider algorithms for actually solving OCSP. The simplest algorithm is the brute-force algorithm: first collect all values from all information sources, and then run an efficient CSP solver to generate a solution to the problem. However, this can be very costly. 
It is clearly preferable to only gather information as needed, as shown by function o-search(Algorithm 1). If there is a solution within the values available from the

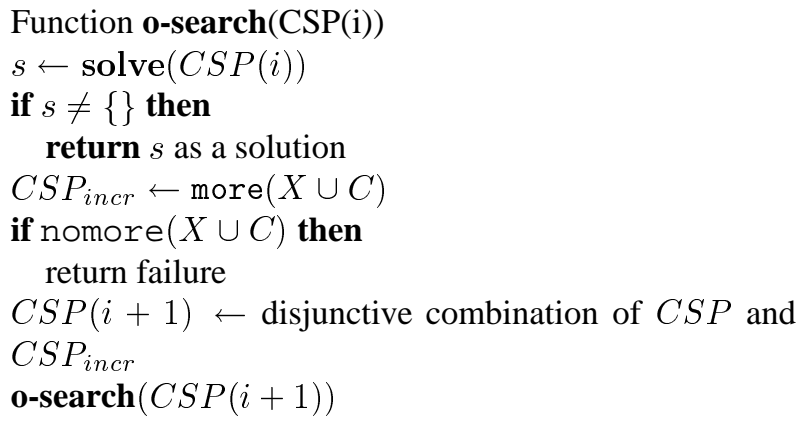

Algorithm 1: o-search: an incremental algorithm for solving OCSP.

servers, this algorithm will eventually find it, since the mediator will eventually return every value or value combination that the servers can provide. While it is already quite efficient, it can be improved upon by focussing information gathering on the parts of the CSP that caused the failure.

\subsection{Focusing information gathering on conflicts}

New values have to be gathered only when the current instance $C S P(i)$ has no solution. In that case, it usually contains a smaller subproblem that already has no solution, and $C S P(i)$ can be made solvable only by creating a solution to that subproblem. Information gathering thus should focus on the variables in the subproblem.

The following proposition provides the basis for identifying variables that are part of unsolvable subproblems:

Proposition 1 Let a CSP be explored by a failed backtrack search algorithm with static variable ordering $\left(x_{1}, \ldots, x_{n}\right)$, and let $x_{k}$ be the deepest node reached in the search with inconsistency detected at $x_{k}$. Then $x_{k}$, called the failed variable, is part of every unsolvable subproblem of the CSP involving variables in the set $\left\{x_{1} . . x_{k}\right\}$.

Proof: see [17].

On the basis of this proposition, we can use the results of a failed CSP search process to determine for which variable additional values should be collected.

These are then passed to the mediator, which will search for relevant information on the network. When there are no additional values for this variable, the mediator returns a nomore message, and other variables are then considered.

The resulting algorithm fo-search (failure-driven open search) is shown in Algorithm 2. It makes the assumption that variables are ordered by the index $i$, and uses the array

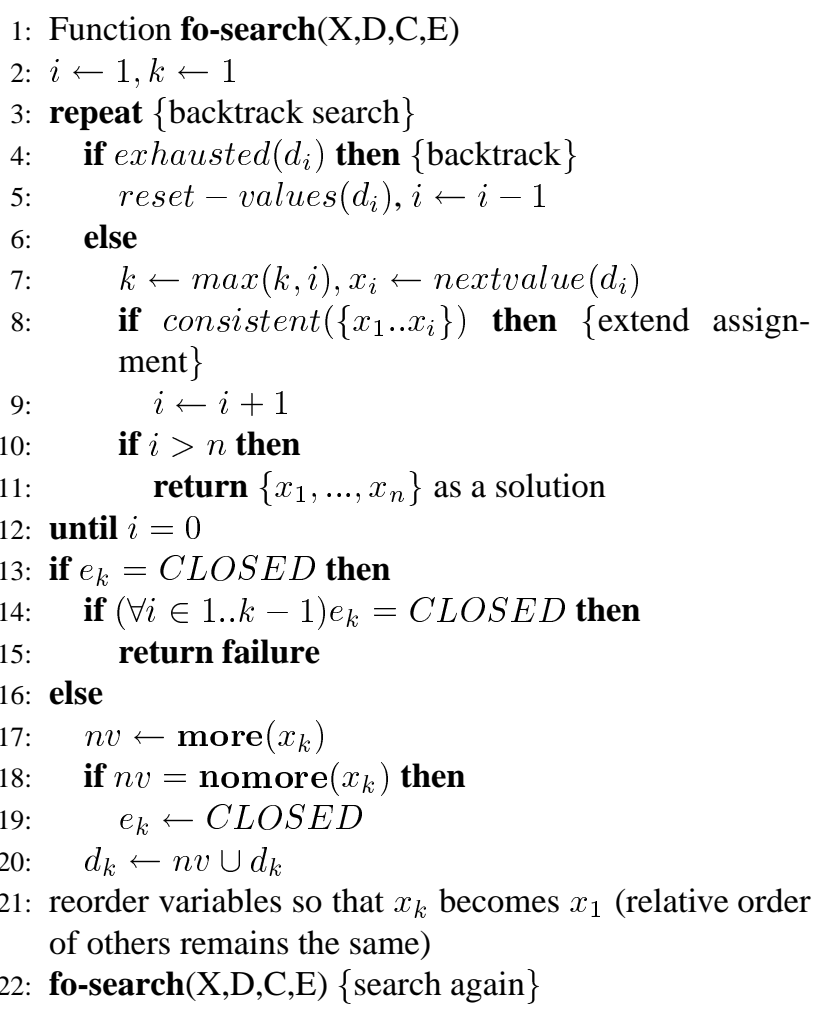

Algorithm 2: Function fo-search for solving OCSP.

$E=\left\{e_{1}, . ., e_{n}\right\}$ to indicate whether the domain for the corresponding variable is completely known (CLOSED). The algorithm assumes that no constraint propagation is used, although the chronological backtracking can be replaced with backjumping techniques (jumping directly to the last constraint violation) to make it more efficient.

In [17], we show that if the current instance $C S P(i)$ contains a minimal unsolvable subproblem, Algorithm 2 will either solve it or fall into a mode where it cycles through the variables in this minimal subproblem and gathers values for each of them. This allows allows showing that the algorithm is complete even in the presence of unbounded domains.

For efficiency reasons, it may be advantageous for the mediator to obtain values not only for single variables, but entire subproblems with a single query. Algorithm 2 can be modified for this case in two ways:

- it can iterate without additional queries until a minimal subproblem is completely identified, and then call the mediator on that subproblem or subproblems that have a maximal overlap with that subproblem.

- it can gather additional values for all subproblems that include the last failed variable. This would preserve completeness of the algorithm, but may be less efficient 


\section{Extension to Fuzzy Optimization Problems}

We can extend the open constraint programming context to optimization problems by adding to each variable domain a valuation function that indicates the cost of the corresponding value. Thus, we define:

Definition 3 An open constraint optimization problem $(O C O P)$ is a possibly unbounded, partially ordered set $(\mathrm{COP}(0), \mathrm{COP}(1), \ldots)$ of constraint satisfaction problems, where $C O P(i)$ is defined by a tuple $\langle X, D(i), W(i), C>$ where

- $X, D(i)$ and $C$ are as in Definition 1.

- $W(i)=\left\{w_{1}(i), w_{2}(i), \ldots, w_{n}(i)\right\}$ is a set of cost (weight) functions on the domains in $D$, where $w_{i}$ : $d_{i} \rightarrow \Re^{+}$gives the cost associated with each value in the domain $d_{i}$. Costs are normalized so that $\min _{d \in d_{i}} w_{i}(d)=0$.

An assignment is optimal for an instance COP(i) if it is consistent and there is no other consistent assignment of $C O P(i)$ such that the combination of the weights of all values $w_{j}(i)\left(v\left(x_{j}\right)\right)$ is better.

An assignment is the solution to an instance COP $(i)$ if it is optimal and there is no other optimal assignment that is lexicographically smaller.

An assignment is the solution to an $O C O P(i)$ if it is the solution for an instance $\operatorname{COP}(i)$ and optimal for all instances $\operatorname{COP}(j), i<j$, and there is no other assignment which is optimal for an instance $\operatorname{COP}(k), k<i$ and also optimal for $C O P(i)$.

Again, the optimization problem may have to be transformed using hidden variable encoding.

In order to make it possible to guarantee optimality, we need to introduce the following, very important assumption:

Assumption: information sources always return values in non-increasing order of weight, i.e. they return the most preferred values first.

Different formulations of the optimization problem exist depending on how the weights of the individual variable assignments are combined. There are two major classes of combination functions:

- in weighted COP, the sum of the weights is minimized. This applies for example when weights represent the costs of choosing a value.

- in fuzzy COP, the maximum of the weights is minimized. This applies for example when the weights represent the delay that will be entailed by choosing a value. We note that our definition has opposite parity as classical fuzzy logic: a weight of 0 is best and 1 (or a higher number) is worst.
Algorithms for solving OCOP problems with the weighted model are very different from the OCSP algorithm described earlier and so we do not treat them in this paper. Algorithms for solving the weighted model as discussed in [18]. However, it is possible to generalize Algorithm 2 to the case of fuzzy COP.

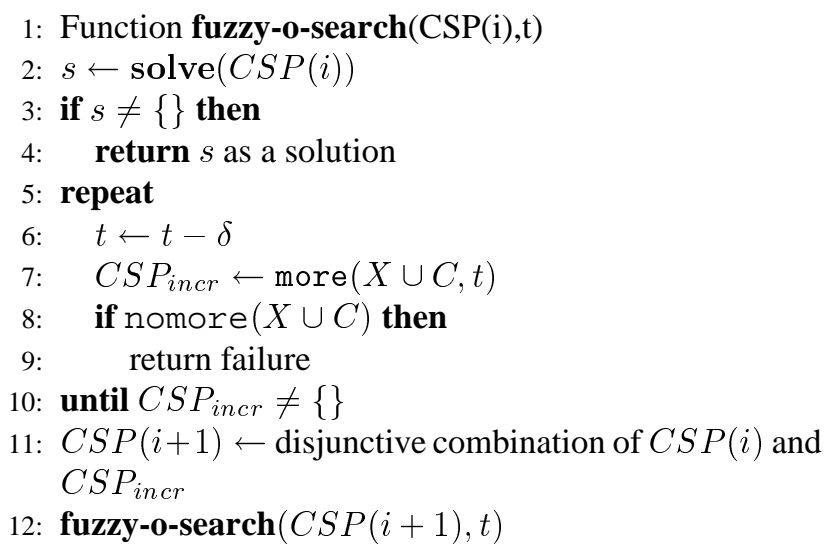

Algorithm 3: fuzzy-o-search: an incremental algorithm for solving OCSP.

In fuzzy constraint optimization, the goal is to minimize the maximum weight of any constraint. Any constraint satisfaction algorithm can be turned into an optimization algorithm for fuzzy CSP by introducing a threshold $t$ for this maximum cost. Starting with the lowest possible threshold (0), it can be gradually increased until the problem becomes solvable. Algorithm 3 shows this adaptation for Algorithm 1. The algorithm assumes that by adding the threshold as an additional argument to the function more, the mediator will return all values with valuation up to that threshold. The algorithm should initially be called with the lowest possible threshold, i.e., $t=0$. It is easy to show that:

Theorem 1 For sufficiently small increment $\delta$, Algorithm 3 will find the optimal solution.

Proof: The solution found by the algorithm does not contain any values with valuations worse than the final value of $t$, so its valuation is not worse than $t$. Now assume that there was a solution with valuation better than $t-\delta$. Then the algorithm would have found this solution on the previous iteration. Thus, if $\delta$ is sufficiently small, Algorithm 3 will return the optimal solution.

However, this algorithm is not necessarily optimal with respect to the number of values queried. To minimize the number of queries to information sources, we have to consider what it means for a consistent assignment of values to the variables to be optimal for a fuzzy COP: 
Proposition 2 An assignment to an instance $C O P(i)$ of a fuzzy OCOP with maximum weight $t^{*}$ is optimal for all higher instances $C O P(j), C O P(i) \prec C O P(j)$ if either there is a subproblem whose optimal weight is $t^{*}$, or all domains have been generated completely or up to at least one value with weight $\geq t^{*}$, and there is no consistent assignment with lower fuzzy cost than $t^{*}$.

\section{1: Function fuzzy-fo-search $(\mathrm{X}, \mathrm{C}, \mathrm{D}, \mathrm{W}, \mathrm{E}, \mathrm{t})$}

$i \leftarrow 1 ; k \leftarrow 1$

repeat $\{$ backtrack search $\}$

if exhausted $\left(d_{i}\right)$ then $\{$ backtrack $\}$

reset - values $\left(d_{i}\right), i \leftarrow i-1$

else

$k \leftarrow \max (k, i), x_{i} \leftarrow$ nextvalue $\left(d_{i}\right)$

if consistent $\left(\left\{x_{1} \ldots x_{i}\right\}\right) \wedge\left(\max _{i=1 . . i} w_{i}\left(x_{i}\right) \leq t\right)$

then $\{$ extend assignment $\}$

$$
i \leftarrow i+1
$$

if $i>n$ then

return $\left(\left\{x_{1}, \ldots, x_{n}\right\}\right)$ as a solution

until $i=0$

if $e_{k}=C L O S E D$ then

if $(\forall i \in 1 . . k-1) e_{k}=$ closed then

return failure

else

if $e_{k} \neq M A R K E D$ then

$e_{k} \leftarrow M A R K E D$

else

$\operatorname{For}(i \in 1 . . k)$

if $\left(e_{i}=M A R K E D\right) e_{i} \leftarrow U N M A R K E D$

$u \leftarrow 1$

repeat

$j \leftarrow \operatorname{argmin}_{i \in 1 . . k, e_{i} \neq C \operatorname{LOSED}}\left(\max _{v \in d_{i}} w_{i}(v)\right)$

$(n v, n w) \leftarrow \operatorname{more}\left(x_{j}\right)$

if $n v=$ nomore then

$e_{j} \leftarrow C L O S E D$

else

$d_{j} \leftarrow n v \cup d_{j} ; w_{j} \leftarrow n w \cup w_{j}$

$(a, s) \leftarrow \operatorname{optimize}(X, D, C, R, W)$

if $(a \neq N I L) u \leftarrow s$

until $(\forall i \in 1 . . k)\left[\left(\max _{v \in d_{i}} w_{i}(v) \geq u\right) \vee\left(e_{i}=\right.\right.$ $C L O S E D)]$

33: $t \leftarrow \max (t, u)$

34: reorder variables so that $x_{k}$ becomes $x_{1}$; relative orders of others unchanged

35: fuzzy-fo-search $(\mathrm{X}, \mathrm{C}, \mathrm{D}, \mathrm{W}, \mathrm{E}, \mathrm{t})\{$ search again $\}$

Algorithm 4: Function fuzzy-fo-search for fuzzy OCOP.

Proof: The first case is straightforward as a COP cannot be solved unless every subproblem is solved. For the second case, assume that there was an instance $C O P(j)$ that admitted a solution with a better weight $t^{\prime}$. Then this solution would have to use at least one value that is not in $C O P(i)$. As $C O P(i) \prec C O P(j)$, such a value would have to have weight at most $t^{*}$. Since the weight of a solution is the minimum of the weight of the constraints, the weight of the new solution cannot be better than $t^{*}$.

Algorithm 4 is an adaptation of Algorithm 2 that exploits this property to reduce the number of value queries required. Similarly to Algorithm 2, it is driven by failures of solution search. When new values can be queried with valuations better than the current threshold $t$, they are queried immediatly. When the valuation would exceed this threshold, the algorithm does not immediatly query for new values once an unsolvable subproblem is found, but first ensures that it finds a minimal unsolvable subproblem. This is done by first only marking the variables until the same variable is encountered a second time. In this case, it is shown in [17] that the algorithm has found a minimal unsolvable subproblem. It now enters a loop where it systematically increases the domains with the lowest valuations for the variables in this subproblem until it becomes solvable. The threshold is then increased to the valuation of this solution, and henceforth the algorithm allows any solution up to the threshold.

We can show that:

Theorem 2 Algorithm 4 is sound, i.e. the solution it returns is optimal.

Proof: Let the weight of the final solution returned by Algorithm 4 be $t^{*}$. Then the threshold $t$ in the algorithm must reach at least $t^{*}$, for otherwise it would not pass the extension in step 8 . The threshold can only be extended to $t^{*}$ if there is a subproblem whose optimal solution has valuation $t$ and whose domains are all larger than $t$. But then this is a subproblem with optimal cost $t=t^{*}$, and thus the solution with $t=t^{*}$ is optimal for the entire problem as well.

Algorithm 4 may well make more than a minimal number of queries to the information source. In pratice, however, its behavior is close to optimal, similar to that of Algorithm 2 for open CSP.

\section{Experimental results}

\subsection{Constraint Satisfaction experiments}

We tested the performance of the techniques we described on synthetic, randomly generated constraint satisfaction problems. As an example for constraint satisfaction, we use resource allocation (equivalent to list coloring), which can be modelled as a CSP whose variable domains 


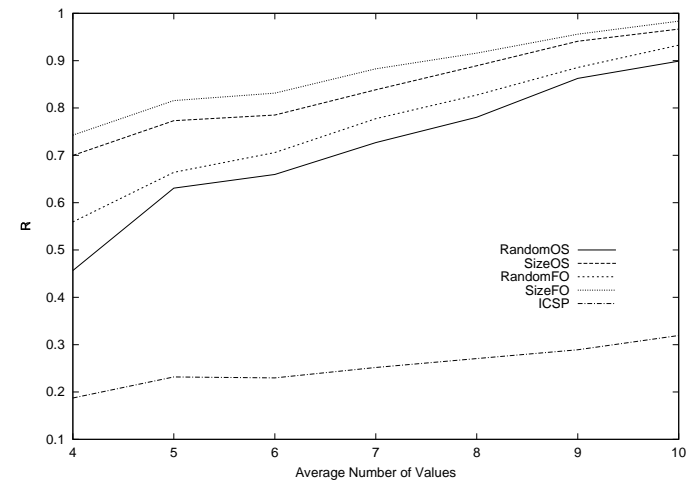

Figure 3. Efficiency ratio against number of values for several combination of search/mediator algorithms.

are resources and whose constraints are all inequalities (expressing the fact that the same resources cannot be used for different tasks at the same time).

We compare the performance of different combinations of algorithms in the mediator and the problem solver. For the mediator, we consider a random algorithm, where information sources are queried in random order, and a size algorithm, where information sources are queried in order of the number of values they are likely to contain. For the problem solver, we consider the two algorithms given earlier, namely $\mathbf{O S}$ for o-search and FO for fo-search.

Several metrics have been developed in the field of database selection ([19]). We measure performance by the ratio:

$R=\frac{\text { Number of variables of the CSP }}{\text { Number of access to IS until a solution is found }}$

Since each variable must have at least one value, solving the CSP requires at least one information source access per variable, so that the ideal value for $\mathrm{R}$ is 1 . Smaller values of $\mathrm{R}$ mean low efficiency. We consider that $\mathrm{R}$ provides a good measure of the relative amount of information gathering effort generated by the two methods, but does not take into account possible parallelization or buffering.

Figure 3 plots the efficiency ratio against the average number of values available for each variable for a setting in which there are a total of 12 information servers. The more values there are for each variable, the easier the problem is to solve, and we can see that the average efficiency in general increases with the number of available values. This is expected as resource allocation gets easier when the number of resources increases.

The best method is, as expected, a combination of failure-driven open search combined with an indexing of the database on size; this gives an efficiency approaching

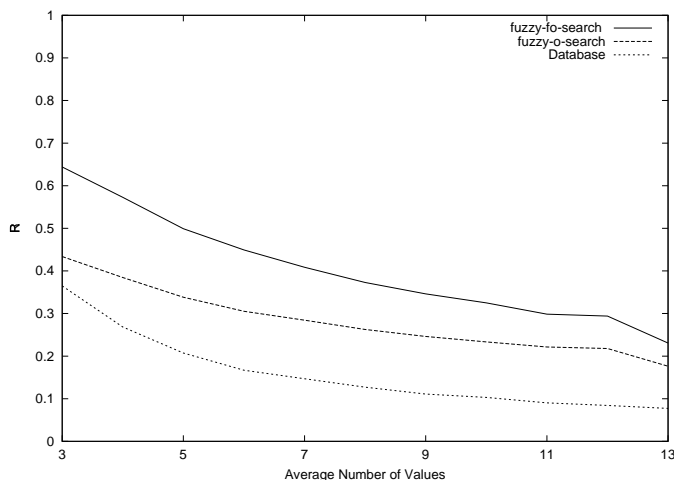

Figure 4. Efficiency ration vs. average number of values for fuzzy OCOP.

the theoretical optimum. It appears furthermore that information about the size of information servers plays a bigger role than directing the search for the right variable, as the next runner-up is the algorithm combining size with opensearch (OS).

The database method of gathering all information beforehand requires gathering all $|D|$ values, resulting in an efficiency ratio of $1 /|D|$. This curve is not shown.

\subsection{Fuzzy OCOP}

Figure 4 shows a comparison of the number of values queried by Algorithms 3 (o-search) and 4 (fo-search) and a database-style method that queries all values beforehand. Since algorithm runtime is a more significant factor, we did not distribute the values in different servers, but only measured the number of values that need to be obtained which provide a similar comparision. Weights were assigned randomly from a uniform probability distribution between 0 and 1 for values and value combinations. The results show a significant reduction in the number of values that need to be obtained when either Algorithm 3 or Algorithm 4 is used. Note in particular that if there were many values with suboptimal cost, as would often be the case in distributed applications, the performance of the database method would be much worse and the relative gains much greater.

\section{Conclusions}

We have investigated how to integrate information gathering in a constraint satisfaction problem solver in order to increase the efficiency of accessing information servers. Assuming an information agent infrastructure that corresponds well to what is envisaged for the semantic web, we have shown experimentally that such integration leads to significant efficiency gains. 
The gains tend to increase with both the number of information servers and the number of values they provide. Thus, the technique is likely to be particularly useful to improve the scalability of intelligent information systems.

On randomly generated resource allocation problems, the number of values that must be gathered is up to orders of magnitude lower than if all values were collected. However, in an environment such as the world wide web, the gains can be much more substantial, as there are many values that can lead to a solution. For example, if the task is to find a consistent combination of hotel, restaurant and theater, search engines would typically return dozens of possibilities for each option, but only a few are needed to find a solution.

In other applications, such as supply chain optimization, every query may itself trigger a problem-solving process to prepare a suitable offer. This will lead to gains multiplying geometrically; for a supply chain of depth 5 a gain of 10 at each stage translates into a factor of $10^{5}$ for the final level.

Even for optimization, gains of about a factor of 3 can be achieved on random problems. However, here we observe a decrease in efficiency as the domains get larger, which is due to the fact than an optimal solution must be guaranteed.

This shows the promise of organizing web-based information systems as networks of agents that provide information on demand rather than attempting to integrate information and apply conventional software solutions.

\section{References}

[1] Genesereth, M. R., Keller, A. M., Duschka, O.: "Infomaster: An Information Integration System", Proceedings of 1997 ACM SIGMOD Conference, May 1997.

[2] Marian Nodine, Jerry Fowler, Tomasz Ksiezyk, Brad Perry, Malcolm Taylor and Amy Unruh: "Active Information Gathering in InfoSleuth," International Journal of Cooperative Information Systems 9:1/2, 2000, pp. 3-28.

[3] A. Sheth and J.A. Larson: "Federated Database Systems," ACM Computing Surveys 22(3), 1990

[4] S. Chawathe, H. Garcia Molina, J. Hammer, K.Ireland, Y. Papakostantinou, J. Ullman and J. Widom: The TSIMMIS project: Integration of heterogeneous information sources. In IPSJ Conference, Tokyo, Japan, 1994

[5] José Luis Ambite and Craig Knoblock: "Flexible and scalable cost-based query planning in mediators: A transformational approach," Artificial Intelligence 118, pp. $115-161,2000$

[6] Alon Y. Levy, Anand Rajaraman , Joann J. Ordille: "Querying Heterogeneous Information Sources Using Source Descriptions," Proceedings of the 22nd VLDB Conference, Bombay, India, 1996
[7] Keith Decker, Katia Sycara and Mike Williamson: "Middle-Agents for the Internet," Proceedings of the 15th IJCAI, Morgan Kaufmann, 1997, pp. 578-583

[8] Katia Sycara, Seth Widoff, Matthias Klusch and Jianguo Lu: "LARKS: Dynamic Matchmaking Among Heterogeneous Software Agents in Cyberspace." Autonomous Agents and Multi-Agent Systems, 5, 173-203, 2002.

[9] Peter M.D. Gray, Suzanne M. Embury, Kit Y. Hui, Graham J.L. Kemp: "The Evolving Role of Constraints in the Functional Data Model", Journal of Intelligent Information Systems 12, pp. 113-137, 1999.

[10] J. Budzik, S. Bradshaw, X. Fu, and K. Hammond: "Supporting Online Resource Discovery in the Context of Ongoing Tasks with Proactive Assistants," International Journal of Human-Computer Studies 56(1) Jan 2002, pp. 47-74

[11] B.J. Rhodes and P. Maes: "Just-in-time information retrieval agents," IBM Systems Journal 39, pp. 685-704, 2000

[12] Christian Bessière: "Arc-Consistency in Dynamic Constraint Satisfaction Problems," Proceedings of the 9th National Conference of the AAAI, pp. 221-226, 1991

[13] Makoto Yokoo: "Algorithms for Distributed Constraint Satisfaction: A Review," Autonomous Agents and Multi-Agent Systems, Vol.3, No.2, pp.189-212, 2000

[14] Gio Wiederhold and Michael R. Genesereth: "The Conceptual Basis for Mediation Services” IEEE Expert, 12(5), 1997.

[15] F. Rossi, C. Petrie and V. Dhar: "On the equivalence of constraint satisfaction problems," Proceedings of ECAI90, pp. 550-556, 1990

[16] K. Stergiou and T. Walsh: "Encodings of Non-binary Constraint Satisfaction Problems," Proceedings of AAAI99, ppp. 163-168, AAAI Press, 1999

[17] Boi Faltings, Santiago Macho-Gonzalez: "Open Constraint Satisfaction," Principles and Practice of Constraint Programming - CP 2002, Springer Lecture Notes in Computer Science(2470), 2002, pp. 356-370.

[18] Boi Faltings, Santiago Macho-Gonzalez: "Open Constraint Optimization," Principles and Practice of Constraint Programming - CP 2002, Springer Lecture Notes in Computer Science, 2003

[19] James C. Freanch and Allison L. Powell: Metrics for Evaluating Database Selection Techniques. 2000 